\title{
Pulse Active Transform (PAT): A Non-Invertible Transformation with Application to ECG Biometric Authentication
}

\author{
Sairul I Safie, Nurfazira H, Azavitra Z \\ Malaysian Institute of Industrial Technology (MITEC) \\ University Kuala Lumpur \\ Johor Bahru, Johor \\ sairulizwan@mitec.unikl.edu.my
}

\author{
John J Soraghan, and Lykourgos Petropoulakis \\ Centre for Excellence in Signal and Image Processing \\ University of Strathclyde, \\ Glasgow, Scotland \\ \{j.soraghan, akis\}@eee.strath.ac.uk
}

\begin{abstract}
This paper presents a new transformation technique called the Pulse Active transform (PAT). The PAT uses a series of harmonically related periodic triangular waveforms to decompose a signal into a finite set of pulse active features. These features incorporate the signal's information in the pulse active domain, and which are subsequently processed for some desired application. PAT is non-invertible thus ensuring complete security of the original signal source. In this paper PAT is demonstrated on an ECG signal and used for biometric authentication. The new transformation technique is tested on 112 PTB subjects. It is shown in this paper that the new transformation has a superior performance compared to the conventional characteristic based feature extraction methods with additional security to avoid recovery of the original ECG.
\end{abstract}

Keywords- Pulse Active Transform, Electrocardiogram, Biometric Authentication

\section{INTRODUCTION}

Features extracted from any location of a signal represent specific information of that signal. In some cases, these locations generate distinctiveness to discriminate individuals using signals such as electrocardiograms (ECGs) [1-4]. This paper presents a new transformation technique called the Pulse Active Transform (PAT). PAT transforms a signal by considering a relationship between multiple pairs of points in a signal, and recombining this relationship to form the transform signal. Features extracted from the signal locations are separately extracted and are used to generate a new form of signal which includes unique information from different parts of the original signal. Unlike most widely used integral transformation techniques such as Fourier, Laplace or Wavelet transforms, PAT does not require any specific kernel function to transform a signal making the transformed signals hard to be inverted.

The new PAT is demonstrated for secure biometric authentication using an ECG as a signal. When algorithms are used to extract features from a biometric trait, it is important that these algorithms are not prone to invertibility attacks i.e. attacks aimed at recovering the original biometric knowing the feature vectors, algorithm and settings parameters, obtainable from the security system [5]. If this biometric information can be reconstructed, it can no longer serve as a biometric trait and in some cases the health information embedded, as for example in ECGs, is no longer private.

The remainder of this paper is organized as follows. Section II derives the new Pulse Active transformation method (PAT). Implementation of PAT for biometric authentication is shown in section III. Section IV discusses the advantages of the fact that the PAT is non-invertible. Finally section V concludes the paper.

\section{PULSE ACTIVE TRANSFORM}

Consider an arbitrary signal, $y_{I}(t)$ as shown in Figure 1.

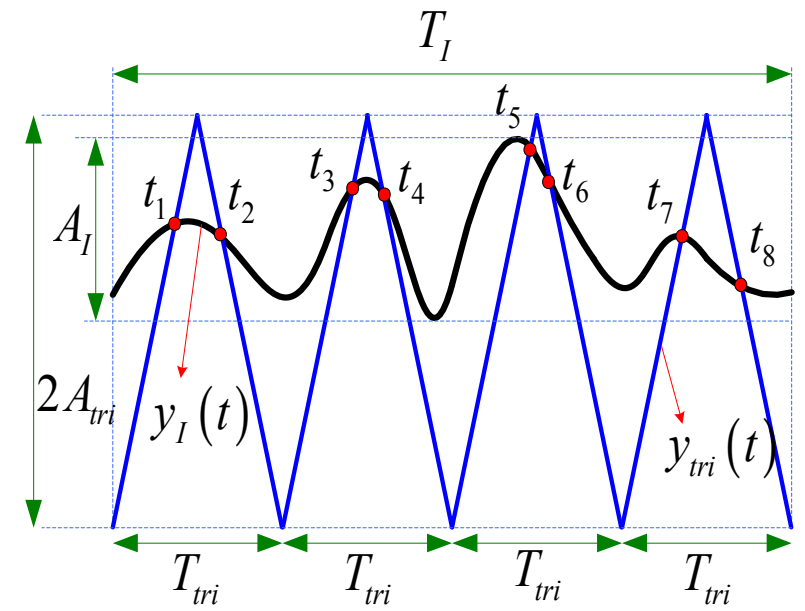

Figure 1: Pulse Active Transformation waveform generation

Let $A_{I}$ and $T_{I}$ be the respective maximum amplitude and signal duration of $y_{I}(t)$. Using PAT the signal under investigation $y_{I}(t)$ is first offset so that its minimum value is equal to zero. A triangular wave, $y_{t r i}(t)$ of a period $T_{t r i}$ with maximum amplitude of $A_{t r i}$ is used to modulate $y_{I}(t)$. Two 
constant parameters known as the integer value modulation factor $m_{f}$, and the modulation index $m_{i}$ are defined as:

$$
\begin{aligned}
& m_{f}=\frac{T_{I}}{T_{t r i}} \\
& m_{i}=\frac{A_{t r i}}{A_{I}}
\end{aligned}
$$

Equations (1) and (2) are used to relate the duration and amplitude between $y_{I}(t)$ and $y_{\text {tri }}(t)$ ensuring that there is an integer number of periods of the triangular waveform contained within $T_{I}$. Hence $m_{f}$ represents the integer number of periodic triangular waves for $y_{t r i}(t)$ used in the interval $T_{I}$. For example in Figure $1, m_{f}$ set equal to 4.

Each period of this periodic triangular waveform intersects the underlying investigated signal at distinct locations. For each triangular wave period, the location of the first intersection from the positive slope $t_{+v e}$ and the location of the last intersection from the negative slope $t_{-v e}$ are selected as the intersection points of interest for that period. Mathematically, this is described as follows:

$$
\begin{aligned}
& t_{(2 m-1)}=t_{+v e} \text { for } \quad(m-1) T_{t r i} \leq t_{(2 m-1)} \leq \frac{(2 m-1)}{2} T_{t r i} \\
& t_{2 m}=t_{-v e} \quad \text { for } \quad \frac{(2 m-1)}{2} T_{t r i} \leq t_{2 m} \leq m T_{t r i} \\
& \boldsymbol{T}_{\text {trans }}=\left[\begin{array}{ll}
t_{(2 m-1)} & t_{2 m}
\end{array}\right]
\end{aligned}
$$

for $m=1,2,3 \ldots m_{f}$

$\boldsymbol{T}_{\text {trans }}$ which is defined as the transition state vector of PAT corresponds to specific intersection location times, as illustrated in Figure 1. To ensure intersections occur within each triangular period, $A_{t r i}$ should be set slightly higher than $A_{I}$. This can be achieved by ensuring $m_{i}>1$.

The PAT $F_{P A}\left[m_{f}\right]$, is calculated by taking the cumulative duration between two contiguous points of $\boldsymbol{T}_{\text {trans }}$ for all $m_{f}$ values. Mathematically, the PAT is calculated as follows:

$$
F_{P A}\left[m_{f}\right]=\sum_{m=1}^{m_{f}}\left(t_{2 m}-t_{2 m-1}\right)
$$

for $m_{f}=1,2,3, \ldots ., M$ where $M$ is the length of the PAT signal. From (6), the first point of the PAT signal $F_{P A}[1]$, is the duration between the intersection points of $y_{E C G}(t)$ and $y_{t r i}(t)$ when a single periodic triangular wave is consider. The next point of the PAT signal $F_{P A}[2]$ is calculated when two periodic triangular waves is used to intersect the ECG complex. This process continues until $m_{f}$ reach the final value of $M$ which can be fixed or set by the user.
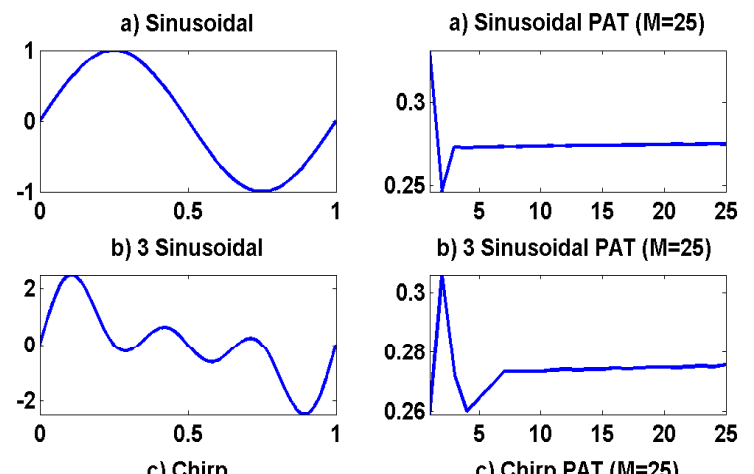

b) 3 Sinusoidal PAT ( $M=25$ )

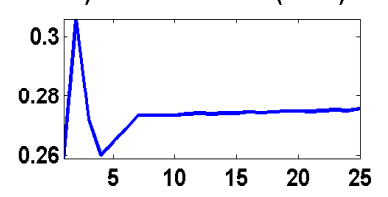

c) Chirp PAT (M=25)
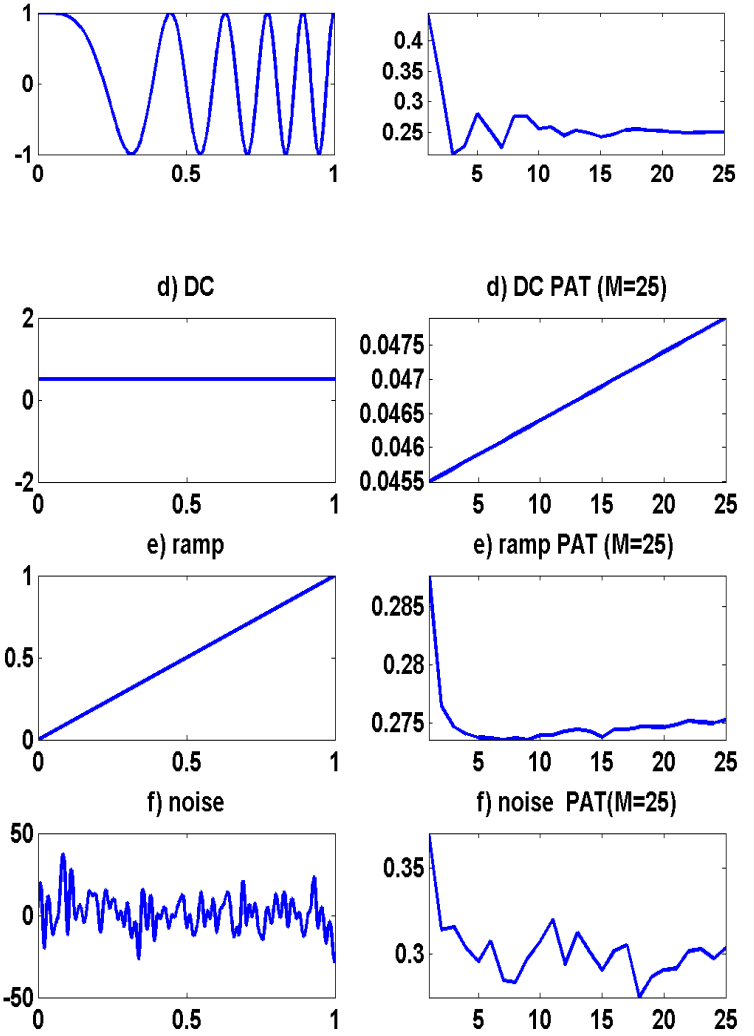

Figure 2: Examples of PA transformed signal

Figure 2 illustrates examples of the PAT on 5 different signals, which include a, (a) single cycle of sinusoidal wave, (b) combination of sinusoidal wave with 3 different frequencies, (c) a chirp (d) DC (e), a ramp signal, and. (f) a random noise. All signals are sampled using $10 \mathrm{kHz}$ frequency. Without loss of generality, the duration of all 
signals is normalized to 1 . These signals are transformed using $M=25$ and the results illustrated in Figure 2 a) to f).

PAT transforms the rate of changes between the duration and amplitudes of the investigated signals into a unique $M$-dimensional vector that may be used as a specific signature of the signal itself. Furthermore, since the PAT is non-invertible, it keeps the signal under investigation completely secure. In this paper we will investigate the validity of this suggestion by applying the PAT feature extraction process to the challenging problem of ECG biometric authentication [3].

\section{PAT FOR ECG AUTHENTICATION}

To implement PAT for ECG biometric authentication, $A_{I}$ will be replaced by $A_{E C G}$ which is the maximum amplitude of the ECG signal, $y_{E C G}(t)$, while the duration between peaks $\mathrm{P}$ and T, $T_{E C G}$ replaces $T_{I}$ as shown in Figure 3.

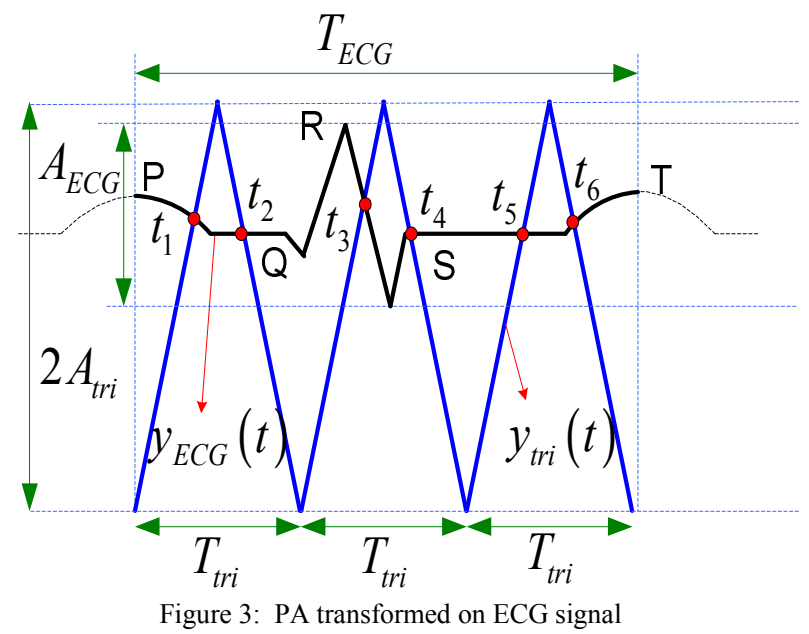

We have described the reasons for selecting this ECG portion in [3]. To validate the performance of the new Pulse Active transforms on ECG biometric system, a database of 112 subjects from the Physikalisch-Technische Bundesanstalt (PTB) database is used [6]. From these 112 subjects, 98 subjects have arrhythmia beats while the rest are healthy. In this paper, biometric performance will be investigated separately between these two populations (healthy and arrhythmia). The PTB database is selected in this study because, for the recordings provided, the average time interval between any two recordings of the same subject is about 500 days [7]. These subjects have at least two different ECG recordings and the ECG sources are taken from a lead I configuration (left and right hand). For each subjects, both recordings will undergo the Pulse Active transformation process. One of these transforms signals will be used for the training database while another will be used for the test. Each of the transforms signal in the training database is compared with all transforms signal in the test database.

The Euclidean distance is used as the distance measure to generate matching scores. If the transformed signals from a test and storage database are from the same subject, the matching score is labeled as a genuine score, else, the matching score is labeled as an imposter score. The score vectors are used to construct the receiver operating characteristic (ROC) curve. The ROC curve plot, is a function of the decision threshold, which plots the rate of 'False Acceptance' (i.e. impostor accepted as genuine) on the $\mathrm{x}$-axis, against the '1False Rejection' (i.e. genuine accepted as genuine) on the yaxis. The area under an ROC curve (AUR) and Equal Error Rate (EER) are the common quantitative measure for comparing ROC curves. Their value ranges between 0 and 1. A higher value of AUR and lower value of EER indicates a system with a better performance.
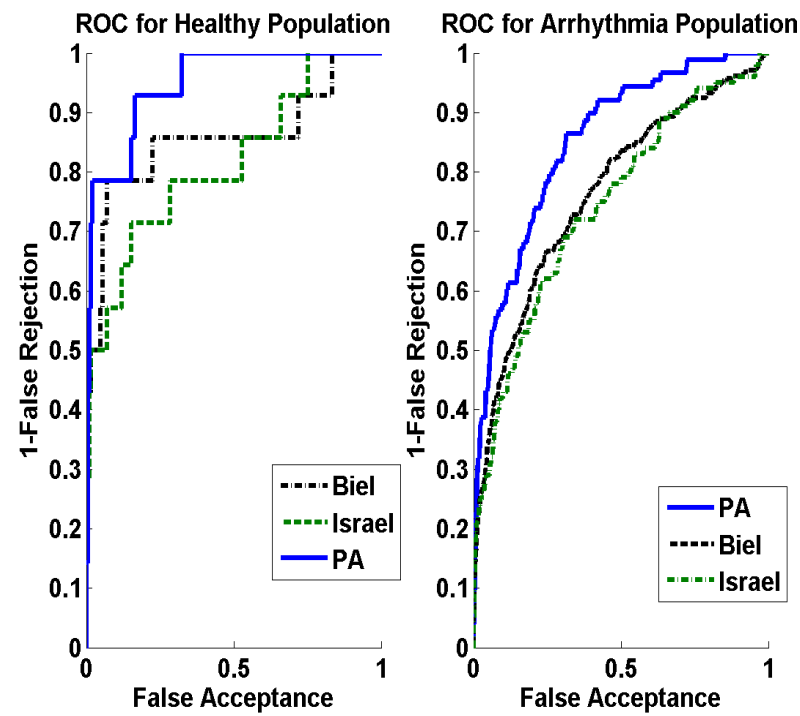

Figure 4: ROC performance curve

Figure 4 shows a comparison ROC curve between the PAT method, Biel's [1] and Israel's [2] feature extraction techniques for healthy and arrhythmia populations. is set to 50 in these experiments. It is clear from both figures in Figure 4 that PAT produces a superior performance compared to the other two methods for both healthy and arrhythmia populations with approximately $9 \%$ improvement in AUR profile and reduction of $7 \%$ in EER values. The AUR and EER values for these techniques are in Table I. 
Table I: Biometric performance comparison values of Figure 4

\begin{tabular}{|l|c|c|c|}
\hline & Pulse Active & Biel [1] & Israel [2] \\
\hline AUR Healthy & 0.9470 & 0.8544 & 0.8136 \\
\hline AUR Arrhythmia & 0.8513 & 0.7630 & 0.742 \\
\hline EER Healthy & 0.1538 & 0.2198 & 0.2857 \\
\hline EER Arrhythmia & 0.2388 & 0.3047 & 0.3101 \\
\hline
\end{tabular}

\section{DISCUSSION}

In the previous section, it was shown that PAT offers superior performance compared to other traditional characteristic based feature extraction techniques for the experimental study. This superior performance reflects the difference between amplitude and morphological shape of the transformed signal as shown in section II.

In ECG biometric technology, it is impossible for two different ECG signals to have the exact same shape even though both ECGs may come from the same subject. This is because ECG signals are very sensitive to heart rate variability (HRV) and electrode placement. These factors will alter the temporal duration and amplitude of the ECG signals.
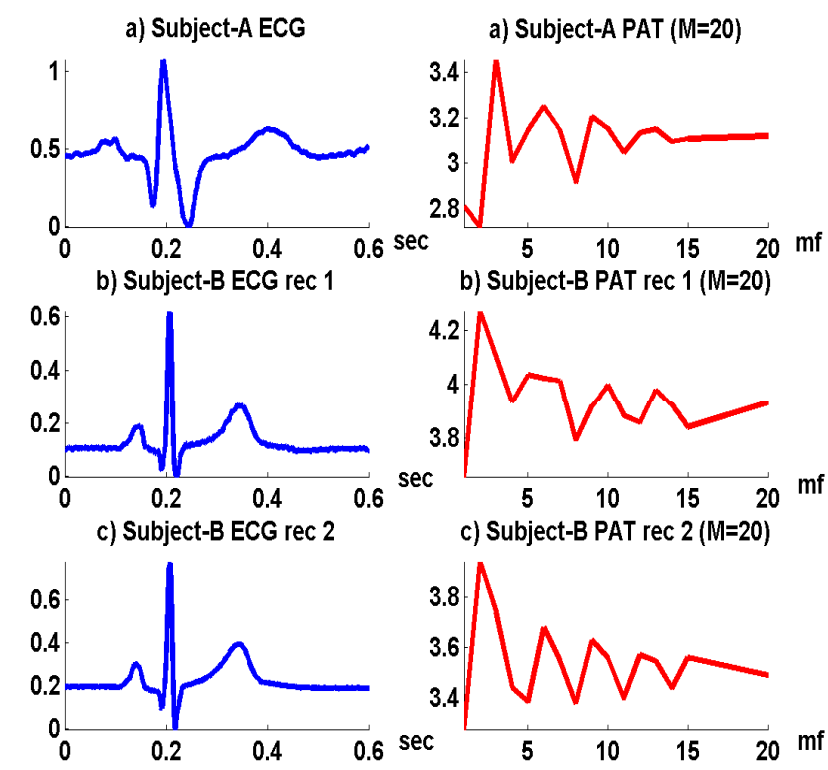

Figure 5: Pulse Active Transformation for on ECG signals

For example, it is known that when the heart rate increases, the total duration for all ECG complexes become shorter. Similarly, when an ECG electrode is placed at a location with thicker skin due, for example, to thicker subcutaneous layer, the signal amplitude of this ECG will be reduced. Figure 5 a) to c) illustrate different ECG signals from 2 subjects being transformed using PAT with $M=20$. As can be seen from Figure $5 \mathrm{~b}$ ) and c), the original and transformed signals of subject B are particularly similar between recordings 1 and 2 . However it is observed that the transformed signals between subjects A and B are particularly different in term of shapes and amplitudes.

The PAT technique offers a solution to the HRV and electrode placement problems by using the parameters $m_{f}$ and $m_{i}$. This is done by first detecting the fiducial points (points of interest which correspond to peak and boundaries of the 3 major waves in an ECG signal). Two of these fiducial points are selected to start and end the PA transformation process. In this work, the peaks of $\mathrm{P}$ and $\mathrm{T}$ are selected as the two fiducial points. Fiducial point locations always vary according to the heart rate. By fixing the value of $m_{f}$, any changes on the heart rate ( $T_{E C G}$ reduction for example) is compensated by the changes of the triangular period ( $T_{t r i}$ tends to follow the changes). Similarly, when the amplitude $A_{E C G}$ is reduced, for a fixed value of $m_{i}$ (in this study $m_{i}=1.1$ ) the amplitude of the triangular wave $A_{t r i}$ also changes.

The values of $A_{t r i}$ and $T_{t r i}$ are fundamental in determining the value of the transition state vector $\boldsymbol{T}_{\text {trans }}$. They reflect the slope of the triangular wave and determine the location where the triangular wave is to intersect the ECG signals. Although the PAT given in (6) is simple, it is impossible to reconstruct the original signal based only on the information of $m_{f}$ and the transform signal $F_{P A}\left[m_{f}\right]$.

\section{CONCLUSION}

A new non-invertible transformation technique for direct signature extraction known as Pulse Active Transform (PAT) was presented in this paper. The usefulness of the PAT method was demonstrated on an ECG signal for biometric authentication. The biometric performance of the proposed transformation technique was shown to be superior compared to the traditional characteristic based feature extraction technique. The PAT is a general transform applicable to any finite length signal segment in order to directly extract a signature. Using signatures of signals instead of actual signals for ECG based authentication safeguards the original signal from being reconstructed and ensures that health information embedded within the ECG signals stays private to the user. 


\section{REFERENCES}

[1] L. Biel, O. Pettersson and L. Philipson, 'ECG analysis : a new approach in human identification', IEEE Trans on Instrumentation and Measurement, 2001,50(3), pp.808-812.

[2] S. A. Israel, J. M. Irvine and A. Cheng, 'ECG to identify individuals,' Pattern Recognition, 2004, 38(1), pp. 133-142.

[3] Safie, S.I.; Soraghan, J.J.; Petropoulakis, L., "Electrocardiogram (ECG) Biometric Authentication Using Pulse Active Ratio (PAR)," Information Forensics and Security, IEEE Transactions on , vol.6, no.4, pp.1315,1322, Dec. 2011
[4] Sairul I Safie, John J Soraghan, and Lykourgos Petropoulakis, "Pulse Active Bit Feature Extractor".18th International Conference on System, Signals and Image Processing, pp 55-58, June 16-18, 2011.

[5] A. Nagar, K. Nandakumar, A. K. Jain, "Biometric Template Transformation: A Security Analysis",Proc. of SPIE, Electronic Imaging Media Forensics and Security XII, San Jose, Jan. 2010.

[6] Goldberger AL, Amaral LAN and Glass L,. 'PhysioBank, PhysioToolkit, and PhysioNet: Components of a New Research Resource for Complex Physiologic Signals.', Circulation 101(23):e215e220.

[7] G. Wübbeler, M. Stavridis and D. Kreiseler 'Verification of humans using the electrocardiogram', Pattern Recognition Letters, 2007, 28(10), pp. 1172-1175. 